\title{
Coracoacromial ligament subligamentous intensity change on magnetic resonance images
}

\author{
(1) Hatice Tuba Sanal ${ }^{1}$, (1) Mehmet Erşen ${ }^{2}$, (1) Mustafa Taşar ${ }^{1}$ \\ 1 University of Health Sciences Turkey, Gülhane Training and Research Hospital, Department of Radiology, Ankara, Turkey \\ 2Bucak State Hospital, Clinic of Radiology, Burdur, Türkiye
}

\section{Date submitted:}

08.09.2020

Date accepted:

28.01.2021

Online publication date:

15.12.2021

\section{Corresponding Author:}

Hatice Tuba Sanal, M.D., University of Health Sciences Turkey, Gülhane Training and Research Hospital, Department of Radiology, Ankara, Turkey

tubasanal@gmail.com

ORCID:

orcid.org/0000-0003-1899-2813

Keywords: Coracoacromial ligament, shoulder impingement syndrome, coracoacromial arch, subacromial space, supraspinatus fascia

\begin{abstract}
Aims: To describe the finding of edema-like intensity beneath the coracoacromial ligament $(C A L)$ on shoulder magnetic resonance (MR) images of patients referred with shoulder pain and/or with the presumption of rotator cuff pathology.

Methods: A total of seven patients were included in the study group having edema-like intensity beneath the CAL on fluid sensitive sequences. None of the patients had any other additional findings such as effusion in the subacromio-subdeltoid bursa, degeneration of the acromioclavicular joint or the rotator cuff rupture. The images of the patients were obtained using two different scanners of 1,5T (six patients) and 3T (one patient). The thickness of CAL at its midportion was also measured.
\end{abstract}

Results: A total of seven patients were included. There were five women and two men with a mean age of 39,28 years. Four of the patients were referred to imaging with the suspicion of rotator cuff pathology only, one patient for having pain in the shoulder and two having both pain and suspicion for rotator cuff pathology. The best imaging plane for this finding of edemalike intensity beneath the CAL was the sagittal plane. The ligament thickness was measured with a mean value of $0.94 \mathrm{~mm}$ (between $0.66 \mathrm{~mm}-1.32 \mathrm{~mm}$ ).

Conclusions: The edema-like intensity restricted beneath the CAL without thickening of the ligament can go with symptomatology as seen in subacromial impingement syndrome. Hence, it is valuable to look for and concern this intensity even if it is the sole finding on shoulder MR images, as a possible indication for symptomatology.

\section{Introduction}

Painful shoulder is one of the most common musculoskeletal complaints affecting up to $30 \%$ of individuals, which may bring them to various outpatient clinics (1-3). Different degrees of rotator cuff disorders are commonly the main etiology for shoulder pain, where impingement of the cuff plays a pivotal role. As an answer to the question why the rotator cuff impinges, two predominant etiologies, in other words "types" have been described as "intrinsic and extrinsic". Of these, extrinsic impingement is a frequent cause for the patients who seek an orthopedic or physical assessment (4). Extrinsic shoulder impingement, mainly in the form of subacromial impingement, is related to the mechanical compression of the structures forming the boundaries of the subacromial space where the rotator cuff passes thorough. The roof of this space is confined by an osteofibrous arch which is called coracoacromial arch formed by acromion, coracoid, and the coracoacromial ligament (CAL) $(5,6)$. Diminishing the size of this space confined within the coracoacromial arch has the potential to lead the impingement symptom of persistent shoulder pain created when elevating the arm above $70^{\circ}$, forcing the arm above the head, and lying on the symptomatic side $(3,6)$.

The CAL forms a bridge between the acromion and the coracoid processes of the scapula, making a restraint to superior displacement of the humeral head (7). The CAL is blamed to have a role in subacromial impingement syndrome by forming the coracoacromial arch together with the other components as mentioned earlier (8). 
Degeneration of the CAL and spur formation at the attachment site of the acromion are reported to be in the spectrum of subacromial impingement syndrome $(9,10)$. Although the histological, anatomical and biomechanical properties of the CAL in the body of the ligament have been studied before, less pertaining to the imaging features of this part of the ligament when symptomatic is published $(11,12)$.

The aim of this study was to describe the finding of edemalike intensity beneath the CAL on fluid sensitive sequences, which is in continuation with the fascia of the supraspinatus muscle, on shoulder magnetic resonance (MR) images of the patients referred with shoulder pain or with the presumption of rotator cuff pathology. According to our research, this sole imaging finding of edema-like intensity in symptomatic patients has not been described before in the setting of impingement syndrome, hence we aimed to draw attention and discuss the potential clinical implications of this observation with literature.

\section{Methods}

This single center, retrospective study included seven patients who had edema like intensity beneath their CALs. None of the patients had any other additional findings such as effusion in the subacromio-subdeltoid bursa, degeneration of the acromioclavicular joint or the rotator cuff rupture, making the strict exclusion criteria. The referral clinics, clinical prediagnosis and the complaints of the patients were noted from the archiving system of the hospital. The examinations were performed in the period between November 2017 and June 2019. The study was approved by the Non-interventional Research Ethics Committee of University of Health Sciences Turkey (protocol number: 19/300; November 12, 2019).

All MR images were evaluated for the edema-like hyperintensity beneath the CAL by three authors (two authors with more than 15 years of experience and one author with one year of experience). Thirty eight patients having this intensity beneath their CALs were detected at first. However, according to our strict criterion, which was about the edema-like intensity beneath the CAL's being the single and the only imaging finding, we eliminated those cases with extra findings such as subacromio-subdeltoid bursitis, acromioclavicular joint arthrosis and any degree of rotator cuff pathology.

The MR images of six patients using 1.5T (Philips-InteraNetherlands) and 1 patient using a 3T (Philips, Achieva, Netherlands) scanner were analyzed. For fluid sensitive images on 1,5 $\mathrm{T}$, the acquired sequences included a combination of T2-weighted spectral presaturation with inversion recovery (SPIR) (TR range/TE range, 1392-1741/60 $\mathrm{ms}$ ) in the oblique coronal and transverse planes, and proton density (PD) SPIR (2567-3225/30 ms) in the sagittal plane. On 3T, the acquired sequences were T2-weighted SPAIR (SPectral Attenuated Inversion Recovery) (TR range/TE range, 3563-4114/60 ms) in the oblique coronal and transverse planes, and PD SPAIR (2657/30 ms) in the sagittal plane.

Since depicting the presence of edema-like intensity beneath the CAL was forming the main purpose of the study, where this can only be carried on fluid sensitive sequences as described above, T1-weighted images were not evaluated for edema intensity. The CAL was tracked along its course from coracoid to acromion and the thickness was measured in the mid portion of the ligament.

\section{Results}

The study included seven shoulders of a patient group composed of five women and two men with age ranging from 21 to 52 years (mean age 39.3 years), only having edema-like intensity beneath the CAL. The characteristics of the subjects are summarized in the Table 1. Five of the patients were referred from the orthopedics, one from physical therapy and one from neurosurgery clinics. Four of the patients were referred with the suspicion of rotator cuff pathology only, one patient for having pain in the shoulder and two having both pain and suspicion for rotator cuff pathology.

The best imaging plane for this finding of edema-like intensity beneath the CAL was the sagittal plane; however, in all planes, the hyperintensity could be observed on fluid sensitive sequences. In these sequences, all of the patients were displaying increased signal intensity beneath the $C A L$, in a triangular space with the $\mathrm{CAL}$ forming superior, the coracohumeral ligament anteriorinferior and the fascia of the supraspinatus muscle the posterior borders (Figures 1, 2, 3). The ligament thickness was measured with a mean value of $0.94 \mathrm{~mm}$ (between $0.66 \mathrm{~mm}-1.32 \mathrm{~mm}$ ) at its mid portion.

\section{Discussion}

The edema-like intensity beneath the CAL observed in our study group may be the first sign in the initiation of subacromial impingement syndrome related to this ligament. Shoulder pain is

\begin{tabular}{|c|c|c|c|}
\hline Case\# & Gender/age & Referred for & Referral clinics \\
\hline 1 & $\mathrm{M} / 21$ & $\mathrm{RCL}$ & Physical therapy \\
\hline 2 & $\mathrm{~F} / 42$ & $\mathrm{RCL}$ & Orthopedics \\
\hline 3 & $\mathrm{M} / 52$ & Pain, RCL & Orthopedics \\
\hline 4 & $F / 28$ & $\mathrm{RCL}$ & Orthopedics \\
\hline 5 & $\mathrm{~F} / 44$ & Pain, RCL & Orthopedics \\
\hline 6 & $\mathrm{~F} / 42$ & $\begin{array}{l}\text { Pain both in } \\
\text { shoulder and } \\
\text { neck }\end{array}$ & Neurosurgery \\
\hline 7 & $\mathrm{~F} / 46$ & $\mathrm{RCL}$ & Orthopedics \\
\hline
\end{tabular}



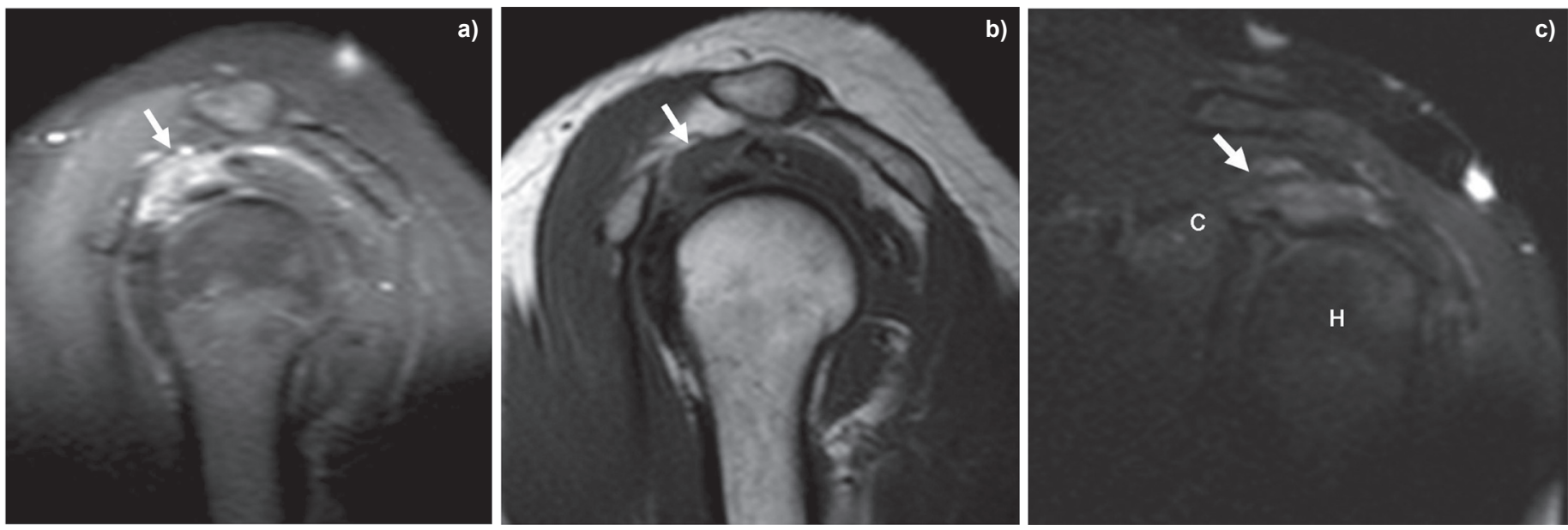

Figure 1. Case \#7, 46-year-old female, referred with the suspicion of rotator cuff lesion. Sagittal proton density SPIR (a), sagittal T1-weighted (b) and oblique coronal T2-weighted SPIR images (c). Edema-like intensity beneath the CAL (arrow) is observed on fluid sensitive sequences (a and c) $\mathrm{C}$ : Coracoid process, $\mathrm{H}$ : Humeral head
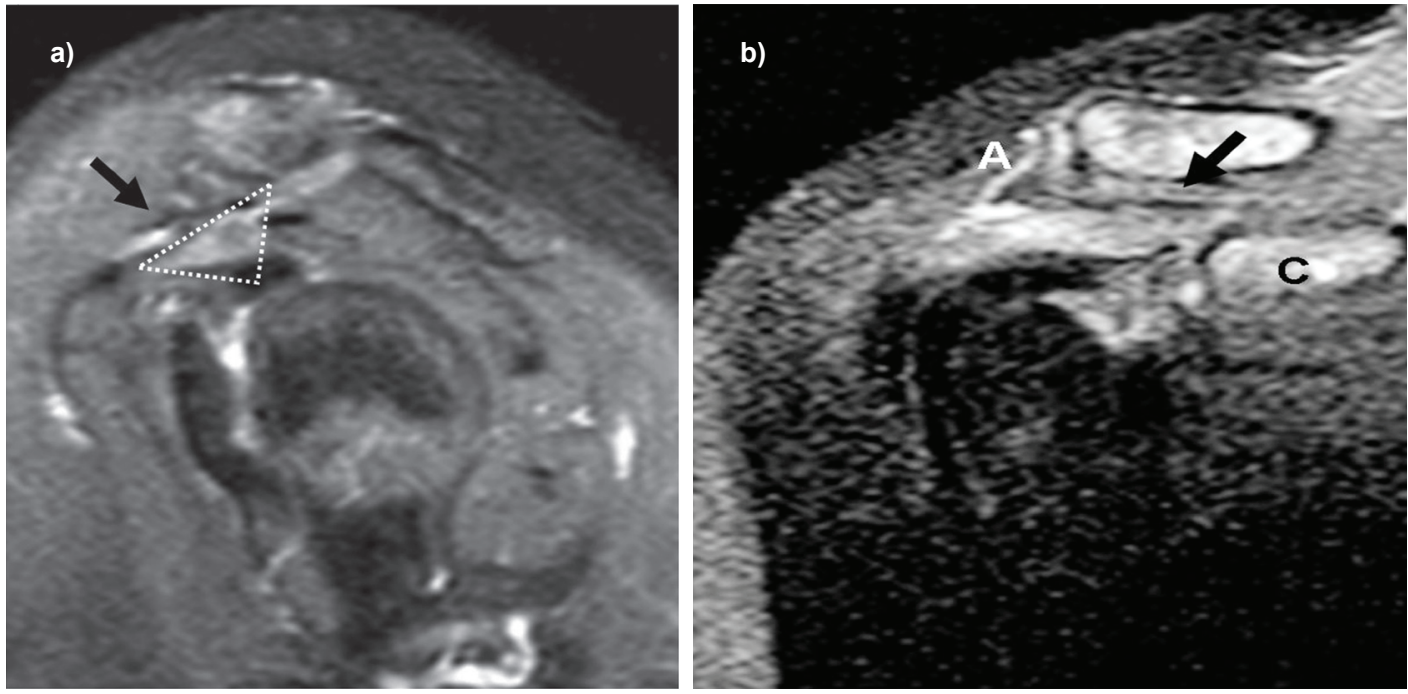

Figure 2. Case \#3, 52-year-old male, referred with the suspicion of rotator cuff lesion with pain. Sagittal proton density SPIR (a), and oblique coronal T2-weighted SPIR images (b). Edema-like intensity beneath the coracoacromial ligament (CAL) (arrow) is seen in a triangle, borders formed by the CAL, the coracohumeral ligament and supraspinatus muscle fascia

C: Coracoid process, A: Acromion

a debilitating condition in which shoulder impingement syndrome is the most commonly reported disorder caused by a myriad of factors. Shoulder impingement syndrome is a compilation of symptoms and signs caused either by pathologies originating within the rotator cuff tendon substances itself (intrinsic) or structures external to the cuff (extrinsic), where occasionally both intrinsic and extrinsic pathologies may coexist (13). According to Neer (14), who described the extrinsic impingement concept first, the subacromial space, where impingement can occur, is boundaried inferiorly by the humeral head and superiorly by the coracoacromial arch which latter encompasses three structures: the undersurface of the anterior third of the acromion, CAL and the coracoid process (3).
The CAL lying between the two parts of the same bone -the scapula- acts as a tension band for the acromion and the coracoid with a significant role in transmitting forces from the surrounding musculature (8). This ligament forms the boundaries of the subacromial space with the humeral head, the acromioclavicular joint and the anterior edge and under surface of the anterior third of the acromion (14). The CAL is also an integrated part of the coracoacromial arch with the anterior third of the coracoid process, the acromion, the distal clavicle and the acromioclavicular joint (15). Narrowing of the subacromial space is one of the mechanistic theories explained for the source of subacromial impingement syndrome (16). When thickened, the CAL may diminish the space for rotator cuff tendon movements (16). A cadaveric study has demonstrated that in certain forceful 

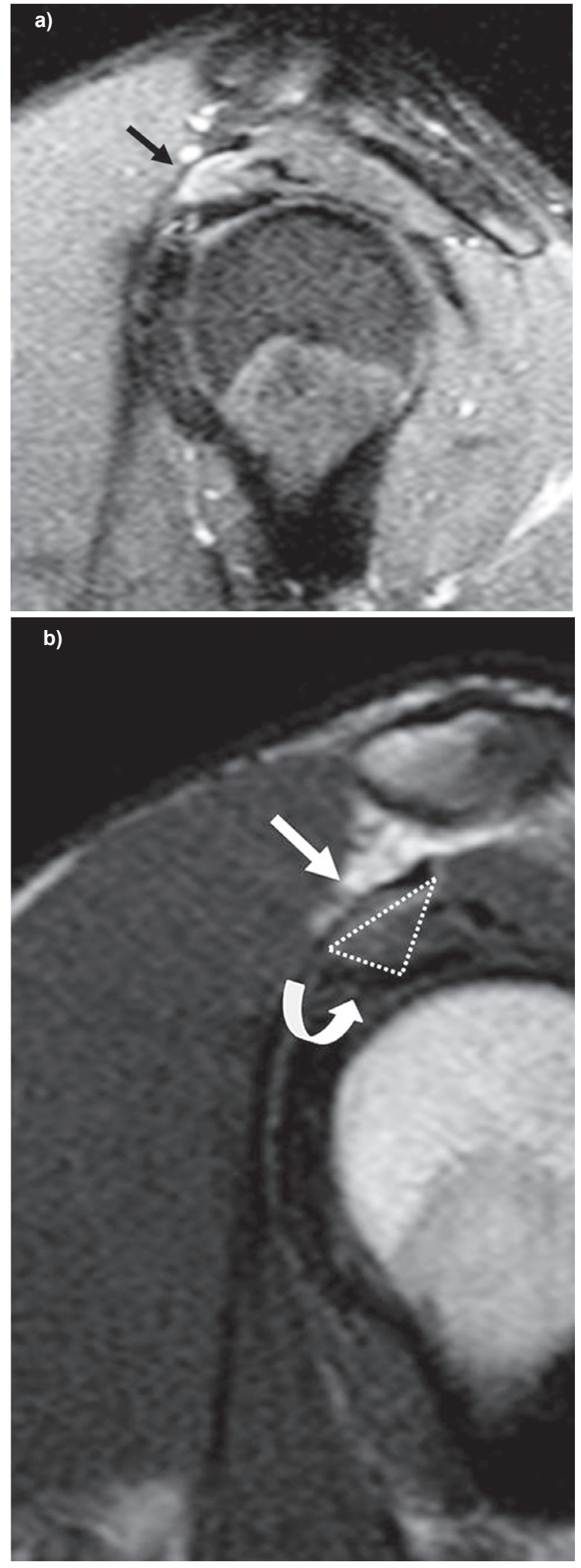

Figure 3. Case \#1, 21-year-old male, referred with the suspicion of rotator cuff lesion. Sagittal proton density SPIR (a), and sagittal T1-weighted (b) images. On fluid sensitive sequence (a) the edema-like intensity beneath the coracoacromial ligament (arrow) is seen in a triangular space with anterior inferior border formed by the coracohumeral ligament (bowed arrow) shoulder and body positions, the rotator cuff impinges at the CAL (17). There are other studies reporting a significant relationship between the existence of a thickened CAL and the incidence of rotator cuff tears $(9,11,18)$.

Although juxtaposition between the coracoacromial arch and the rotator cuff has been shown to be a physiologic phenomenon, others have proposed that recurrent contact between the rotator cuff and the CAL may lead to degenerative changes reciprocally (19).

An adipose tissue is described to line the outermost layer of the CAL where it articulates with the subacromial bursa (7). This adipose tissue is reported to be richly innervated by both free and encapsulated receptor nerve endings, possibly contributing to mechanosensory pathways (7). A study, where the control samples composed of human CALs, showed rich perivascular innervation composed of PGP-9.5-containing nerves in the periligamentous tissue (20). The pain experienced in our patient population with edema-like intensity beneath the CAL may be a reflection of this histological finding.

Age-related changes secondary to chronic stress and cellular degradation with thickening and stiffening of the CAL are noted to contribute to a spectrum of impingement syndrome (7). This subligamantous edema-like intensity of the CAL observed in our study may be related or lead to ligamentous changes and thickening of the ligament ultimately, where it is obvious that long term follow-ups of the patients are needed.

According to a histological study with immunoperoxidase dying by Konttinen et al. (21), which was performed on the materials obtained after Neer's (14) acromioplasty in patients with chronic painful rotator cuff impingement syndrome, neither the CAL nor the periligamentous fatty and loose connective tissue displayed compilation of lymphocytes, macrophages, or other inflammatory cells. Hence, the edema-like intensity on fluid sensitive sequence in our study group is thought to be secondary mainly to reactive synovitis and edema, which needs verification by histological studies that sound practically tough. One other proposal is the frictional effect by the ligament itself. We think that, owing to the periligamentous fatty and loose connective tissue's innervation, possible reactive synovitis in the subligamentous portion of the CAL may be the source of pain in this region.

In their study, Sarkar et al. (12) reported that the CAL did not appear to be primarily responsible in the initiating process of impingement, rather it is the strain that is most likely produced by the changes beginning in the soft tissues of the subacromial space, which may be related to our proposal.

The subligamentous edema-like intensity with heterogeneity in the neighborhood fascia of the supraspinatus muscle made us think a close relationship of the CAL with this muscle fascia in such a continuum, which needs to be confirmed by histology 
again. This edema-like intensity in the subligamentous fatty tissue may be alikened with other impingement syndromes around the other joints such as suprapatellar fat pad impingement syndrome about the quadriceps tendon or infrapatellar fat pad impingement syndrome around the ligamentum mucosum (22).

According to our subject group, these patients with the edema-like intensity beneath the CAL-fascial complex may have the same symptomatology with subacromial impingement syndrome. The awareness of such an intensity change about this anatomical unit may have the potential in future for targeted therapies such as needle applications to this particular space.

The best plane to evaluate the CAL on MR images has been reported to be the oblique sagittal plane in previous studies (8). This was also the same for our study that the high intensity change under the low intensity border of the CAL was well appreciated on the sagittal fluid sensitive sequences. The $\mathrm{CAL}$ extension from the coracoid process to the undersurface of the acromion is well studied at the acromion edge as a causative for impingement. However, the imaging information in the midsubsance and the coracoid extension of the CAL is rare in that it is lacking whether the changes about this aspect of the ligament is symptomatic. Our descriptive study raises this suspect in that the periligamentous changes at the midsection of the CAL approaching to the coracoid process may be symptomatic as well.

The normal thickness of the CAL at the mid portion of the ligament was studied by ultrasound before. In their studies, Wang et al. (23) measured $1.97 \pm 0.49 \mathrm{~mm}(1.1-3.2)$ in normal healthy subjects and Dietrich et al. (24) reported $1.4 \pm 0.2 \mathrm{~mm}$ in asymptomatic volunteers. The ligament thickness in our study was measured with a mean value of $0.94 \mathrm{~mm}$ (between 0.66 $\mathrm{mm}$ and $1.32 \mathrm{~mm}$ ), which is not beyond these reported values.

One of the limitations of this study is the lack of long-term follow-up of patients to observe whether this finding has the potential to lead to a structural change in CAL and ultimately to subacromial impingement syndrome. Owing to the strict exclusion criteria, the small number of the group may sound as another limitation; however, this is a descriptive study of a persistent finding, we think that the number of the cases are sufficient.

\section{Conclusion}

Advances in our understanding of the CAL through the histological and anatomical studies with the clinical and imaging perspective may further aid to the conservative treatments of the patients in the future. According to our observation in this small study group, the edema-like intensity restricted beneath the CAL without thickening of the ligament can go with symptomatology as seen in subacromial impingement syndrome, hence it is valuable to look for and concern this intensity change in the fatty tissue beneath the CAL on shoulder MR images even if it is the sole imaging finding.

\section{Ethics}

Ethics Committee Approval: The study was approved by the University of Health Sciences Turkey of Non-interventional Research Ethics Committee (protocol number: 19/300; November 12, 2019).

Informed Consent: Retrospective study.

Peer-review: Externally peer-reviewed.

\section{Authorship Contributions}

Surgical and Medical Practices: H.T.S., M.E., M.T., Concept: H.T.S., M.E., M.T., Design: H.T.S., M.E., M.T., Data Collection or Processing: H.T.S., M.E., M.T., Analysis or Interpretation: H.T.S., M.E., M.T., Literature Search: H.T.S., M.E., M.T., Writing: H.T.S., M.E., M.T.

Conflict of Interest: No conflict of interest was declared by the authors.

Financial Disclosure: The authors declared that this study received no financial support.

\section{References}

1. Luime JJ, Koes BW, Hendriksen IJ, et al. Prevalence and incidence of shoulder pain in the general population; a systematic review. Scand J Rheumatol. 2004;33:73-81.

2. Urwin M, Symmons D, Allison T, et al. Estimating the burden of musculoskeletal disorders in the community: the comparative prevalence of symptoms at different anatomical sites, and the relation to social deprivation. Ann Rheum Dis. 1998;57:649-655.

3. Smith CP, Vassiliou CE, Pack JR, von Borstel D. Shoulder Impingement and Associated MRI Findings. J Am Osteopath Coll Radiol. 2018;7:5-14.

4. Seitz AL, McClure PW, Finucane S, Boardman ND, Michener LA. Mechanisms of rotator cuff tendinopathy: intrinsic, extrinsic, or both? Clin Biomech. (Bristol, Avon) 2011;26:1-12.

5. El-Shewi IEHAF, El Azizy HM, Gadalla AAEFH. Role of dynamic ultrasound versus MRI in diagnosis and assessment of shoulder impingement syndrome. Egypt $\mathrm{J}$ Radiol Nucl Med. 2019;50:1-7.

6. Garving C, Jakob S, Bauer I, Nadjar R, Brunner UH. Impingement Syndrome of the Shoulder. Dtsch Arztebl Int. 2017; 114:765-776.

7. Rothenberg A, Gasbarro G, Chlebeck J, Lin A. The Coracoacromial Ligament: Anatomy, Function, and Clinical Significance. Orthop J Sports Med. 2017;5:2325967117703398.

8. Bencardino JT, Beltran LS. Pain related to rotator cuff abnormalities: MRI findings without clinical significance. J Magn Reson Imaging. 2010;31:1286-1299.

9. Ogata S, Uhthoff HK. Acromial enthesopathy and rotator cuff tear. A radiologic and histologic postmortem 
investigation of the coracoacromial arch. Clin Orthop Relat Res. 1990;39-48.

10. Ozaki J, Fujimoto S, Nakagawa Y, Masuhara K, Tamai S. Tears of the rotator cuff of the shoulder associated with pathological changes in the acromion. A study in cadavera. J Bone Joint Surg Am. 1988;70:1224-1230.

11. Soslowsky LJ, An CH, DeBano CM, Carpenter JE. Coracoacromial ligament: in situ load and viscoelastic properties in rotator cuff disease. Clin Orthop Relat Res. 1996;(330):40-44.

12. Sarkar K, Taine W, Uhthoff HK. The ultrastructure of the coracoacromial ligament in patients with chronic impingement syndrome. Clin Orthop Relat Res. 1990;(254):49-54.

13. Tien JDY, Tan AHC, MBBS. Shoulder Impingement Syndrome, A Common Affliction of the Shoulder: A Comprehensive Review. Proceedings of Singapore Healthcare. 2014;23:297-305.

14. Neer CS. Anterior acromioplasty for the chronic impingement syndrome in the shoulder: a preliminary report. J Bone Joint Surg Am. 1972;54:41-50.

15. Steinbach LS. Rotator cuff disease. In: Steinbach LS, Tirman PFJ, Peterfy CG, Feller JF, editors. Shoulder magnetic resonance imaging. Philadelphia: Lippincott Williams and Wilkins; 1998. p. 99-133.

16. Michener LA, McClure PW, Karduna AR. Anatomical and biomechanical mechanisms of subacromial impingement syndrome. Clin Biomech (Bristol, Avon). 2003;18:369-379.
17. Burns WC, Whipple TL. Anatomic relationships in the shoulder impingement syndrome. Clin Orthop Relat Res. 1993;(294):96-102.

18. Farley TE, Neumann CH, Steinbach LS, Petersen SA. The coracoacromial arch: MR evaluation and correlation with rotator cuff pathology. Skeletal Radiol. 1994;23:641-645.

19. Gallino M, Battiston B, Annaratone G, Terragnoli F. Coracoacromial ligament: a comparative arthroscopic and anatomic study. Arthroscopy. 1995;11:564-567.

20. Santavirta S, Konttinen YT, Nordström D, et al. Immunologic studies of nonunited fractures. Acta Orthop Scand. 1992;63:579-586.

21. Konttinen $\mathrm{YT}$, Santavirta $\mathrm{S}$, Paavolainen $\mathrm{P}$, et al. Immunoreactive neuropeptide nerves in ligamentous tissue in chronic shoulder pain. Arch Orthop Trauma Surg. 1992;111:341-344.

22. Lapègue $\mathrm{F}$, Sans $\mathrm{N}$, Brun $\mathrm{C}$, et al. Imaging of traumatic injury and impingement of anterior knee fat. Diagn Interv Imaging. 2016;97:789-807.

23. Wang YC, Wang HK, Chen WS, Wang TG. Dynamic visualization of the coracoacromial ligament by ultrasound. Ultrasound Med Biol. 2009;35:1242-1248.

24. Dietrich TJ, Jonczy M, Buck FM, Sutter R, Puskas GJ, Pfirrmann CW. Ultrasound of the coracoacromial ligament in asymptomatic volunteers and patients with shoulder impingement. Acta Radiol. 2016;57:971-977. 